\title{
Shear-Induced Amyloid Formation in the Brain: IV. Effects on Synapses Surrounding Senile Plaque and in Plaque-Free Regions
}

\author{
Conrad N. Trumbore* \\ Department of Chemistry and Biochemistry, University of Delaware, Newark, DE, USA
}

Accepted 5 August 2018

\begin{abstract}
Amyloid- $\beta$ oligomers $(\mathrm{A} \beta \mathrm{O})$ have been proposed as neurotoxins in the synaptic dysfunction that precedes Alzheimer's disease symptoms. Human and animal model studies report that senile plaques contain a halo of A $\beta O$ molecules surrounding these plaques. A far smaller number of oligomers are distributed widely in plaque-free regions. It has been suggested that oligomers migrate from halos to nearby synapses and are incorporated into both pre- and postsynaptic terminals. These two types of oligomers have two different toxicities when extracted and injected in animal models. This paper proposes a shear-energy based explanation for the data in these studies. Shear hypotheses in the preceding three papers in this series are applied to suggest how the hydrodynamics and resulting shear patterns explain the spatial distribution of both A $\beta O$ types, the apparent synapse loss in the vicinity of plaque particles, and possible reasons for the differing toxicities. A shear-based mechanism is proposed for the preferential migration of locally shear-excited A $\beta$ molecules into the synaptic cleft. It is proposed that high energy laminar shear generated by the forced diversion of interstitial fluid around the flow-impeding plaque particle is responsible for the formation of $\mathrm{A} \beta \mathrm{Os}$ around the plaque. It is suggested that in plaque-free regions, a different type of $A \beta O$ with different toxicity is generated by lower energy shear flow around synapses, depositing $A \beta O$ within the synapse from either the neuron membrane surface or by prion-like seeding within the synaptic cleft by locally-sheared $A \beta$ molecules near the synapse entry.
\end{abstract}

Keywords: Amyloid, amyloid- $\beta$, oligomers, prion, seeding, shear energy, synapse, synaptic cleft

\section{INTRODUCTION}

Over one hundred years after the first reported case of Alzheimer's disease (AD), there are nearly 50 million worldwide cases of this currently incurable, devastating disease [1]. Although the onset of AD is commonly attributed to "misfolded" amyloid$\beta(A \beta)$ protein molecules, there has been no reported isolation or characterization of this intermediate nor any widely accepted mechanism for how this protein came to be misfolded [2]. In contrast, there has

\footnotetext{
${ }^{*}$ Correspondence to: Conrad N. Trumbore, 441 Crosslands Drive, Kennett Square, PA 19348, USA. Tel.: +1 610388 7093; Fax: +1 610388 5691; E-mail: conradt@udel.edu.
}

been much progress in identifying the complex cellular events that follow the initiating events that lead to misfolded neurotoxic aggregated $A \beta$ oligomer molecules and their interactions within the brain $[1,3]$. The background and justification for focusing on oligomer molecules being the main neurotoxic toxic compounds in contrast with other amyloids such as protofibrils and plaque has been made in previous papers [4-6].

In these three papers in this series [4-6], it is proposed that the comparatively slow flow of interstitial fluid (ISF) through very narrow, extracellular spaces (ECS) within the brain parenchyma generates and transfers relatively low amounts of shear energy to flowing dissolved $A \beta$ molecules. 
This produces mechanically shear-distorted excited molecules $\left(\mathrm{A} \beta^{*}\right)$ with a conformation that is quite different from any that are found in quiescent solutions. There are at least two important published precedents for such a concept. These are based on the differences in the kinetics of formation [7] and the structural differences of amyloid fibrils [8] formed under quiescent versus shearing conditions.

The suggestion has also been made in an earlier paper [4] that the flow of ISF and/or cerebrospinal fluid (CSF) through and/or around the brain vascular system generates shear-energy that results in cerebral amyloid angioplasty (CAA) deposits in the brain artery region. This suggestion and hypotheses in this series of papers are based on our early work with aqueous protein samples passing through very small diameter stainless-steel high performance chromatography connecting capillaries and observations that protein was being deposited on the capillary walls $[5,9]$. We suggested that protein shear at the wall was responsible for this solid deposition. More recently, the suggestion was made that these results may be not only be responsible for protein losses in amyloid chromatography, but also may cause errors in the analysis of CSF from spinal tap samples [5].

In Fig. 6 of our early paper [9], there was a report of a sudden release of wall-adsorbed protein aggregate after repeated injections of protein into a capillary. The tongue-in-cheek comment was made by the author that this was a good example of a "chromatographic heart attack." Nothing in the intervening years since that remark has discouraged this author from contemplating the potentialities for pioneering shear studies in vascular research, especially using the system proposed by the author in the second paper in this series [5].

The author has more recently proposed [6] that a shear-excited $A \beta^{*}$ molecule can contain highly variable amounts of shear energy, potentially with quite different chemical consequences. The amount of energy depends on the local brain shear map. It is proposed that mechanical shear energy can be used to overcome activation energy barriers that prevent a conformational change from a partially helical to the cross beta-pleated sheet structure [10] needed to transform $\mathrm{A} \beta$ molecules into neurotoxic oligomer precursors within the brain parenchyma.

It is suggested that mechanical shear-induced molecular stretching and deformation produced by physical processes within the brain ECS produce amyloid molecular conformations that might only rarely, if ever, be created by random high-energy thermal events in $A \beta$ solutions commonly encountered in quiescent laboratory amyloid experiments.

Thus mechanical-chemical mechanisms have been proposed in papers of this series that justify the critical role of $A \beta$ concentration in the ultimate formation of neurotoxic $A \beta$ oligomers, especially in membranes $[4,6]$. Because laminar shear is at a maximum at confining walls of ISF flowing through very narrow parenchymal spaces, the role of wall shear, involving membrane surfaces of such critical brain constituents as neurons, synapses, astrocytes, microglia, etc., has been incorporated into a mechanism that is different from that proposed to occur in free-flowing ISF (a short distance away from the wall). There are two main types of shear considered in these hypotheses, laminar and extensional. For examples and further discussion on fluid shear in the brain, consult reference $[4,6]$ and the discussion below.

\section{PROBLEMS IN STUDYING OLIGOMERS AND IDENTIFYING CLINICAL TARGETS}

$A \beta$ is the main chemical constituent of a senile plaque. Historically, the plaque was thought to be the toxic substance responsible for the induction of $\mathrm{AD}$, with its formation proposed to arise from a stepwise addition of individual $A \beta$ molecules forming a linear polymer of $A \beta$, the amyloid cascade reaction [11]. However, plaque generation in the brain did not correlate with $\mathrm{AD}$ symptoms. Current theories are now in general agreement that $A \beta$ oligomers, water soluble $A \beta$ molecule aggregates of various sizes and conformations, are the most toxic and pathogenic forms of $A \beta . A \beta_{42}$ naturally forms in vitro oligomers, ultimately forming protofibrils and fibrils. However, there is a complex equilibrium among many of these oligomers, so that it is difficult to isolate and study them. This has led to cross linking as a way of stabilizing these oligomers so that their conformations and other properties may be studied [13]. Many of the manipulations used in analytical procedures and laboratory preparation of solutions involve exposure to shear, so that oligomer formation in some instances may be shear-induced, especially if, as this author has recently proposed [6], shear induces the formation of prion-like oligomer seeds [14].

The author has also warned [6] that shear-induced aggregation during its collection process may lead to faulty analytical results in spinal tap CSF oligomer concentrations measurements that are one of the means of following the progression of AD. Such 
Table 1

\begin{tabular}{ll}
\hline Type1/Peak1 & Type2/Peak2 \\
\hline Toxic & Non toxic \\
High molecular weight $(>50 \mathrm{kDa})$ & Low molecular weight $(<50 \mathrm{kDa})$ \\
Disrupt memory function & Do not disrupt memory function \\
Unrelated to plaques & Temporally, spatially, and structurally related to plaques \\
In vitro: bind cultured synapses & Examples: dimer, trimer $\mathrm{A} \beta$ \\
In vitro: produce reactive oxygen species & \\
Example: $\mathrm{A} \beta * 56(56 \mathrm{kDa})$ & \\
\hline
\end{tabular}

errors are possible because of the liquid shear present at and near the walls of the spinal tap needle, which can cause $A \beta$ aggregation at the inner surface of the needle, giving rise to deposition of aggregated $A \beta$ on the inner surface of the needle. This complication is further complicated by the possible release of aggregated $A \beta$ deposits from the wall of a capillary of similar inner diameter when flow is stopped (Trumbore $\mathrm{CN}$, Paik J, Vachet R, unpublished results). Experiments examining the adsorption and desorption of $A \beta$ and its aggregates from clinical needle walls need to be performed in a clinical setting, as previously urged in reference [5].

For example, it is reported that early in the course of $\mathrm{AD}$, the in vivo concentrations of $\mathrm{A} \beta$ decrease as the oligomer concentrations increase. However, in vivo oligomer concentrations are apparently much higher than the $A \beta$ concentration. In deciding what the clinical target should be for $A D$, because of the shift from the $A \beta$ cascade to oligomers as a major pathological focus, oligomers have become such a target. But which oligomer? There are a large number of oligomers, most of which are toxic, but very few form stable compounds.

The author reminds clinicians that, if shear is an important variable in oligomer formation, then one neglected target has been a common precursor to all oligomer formation. That is the mechanical shearinduced excited state $A \beta *$ proposed [6] to lead to the formation of oligomer seeds [10]. The author recognizes that shear is somewhat foreign to most amyloid researchers, but has proposed a simple experimental system with which to study amyloid solutions under highly variable shear conditions [6] for use in a variety of experimental situations. It is proposed that more study is needed of this critical shearinduced $A \beta^{*}$ molecule and its reactions with other $A \beta$ molecules and $A \beta$ aggregates and in the presence of candidate drugs while under shear conditions.

If shear is important, there is a need to identify in vivo examples of biological situations where shear may be shown to be involved. The author believes the following is one such situation.

\section{A $\beta$ OLIGOMERS, SYNAPSE LOSS, AND MEMORY FUNCTION}

Since the introduction of the A $\beta O$ hypothesis [15], encompassing over 4,000 research papers, in vivo oligomers have been classified in Table 1 broadly into two types [12].

There has been some controversy as to the differences between $\mathrm{AD}$ brain-derived and synthetic oligomers [16]. One explanation for the possible difference given is the possibility of in vivo posttranslational alterations to the $A \beta$ oligomer. Another is that proteins other than $A \beta$ are involved in these oligomers. Because $A \beta$ molecules are certainly not the only proteins in the ISF stream, any shear condition that affects $A \beta$ will also be affecting other dissolved proteins. Thus, it is likely that mixed (A $\beta$ other non-A $\beta$ protein) shear-induced soluble, toxic aggregates could be responsible for the non-canonical $\mathrm{A} \beta$ brain-derived aggregates discussed by Brody et al. [16].

This author adds one other possible explanation for these oligomer differences. There may be shearinduced $\mathrm{A} \beta$ conformational changes leading to dimer seeds that produce altered conformations of brainderived oligomers that are different from synthetic oligomers [6]. However, the amount of shear energy needed to induce such conformation changes may be an important variable and may be different in different regions of the brain.

Prior to the onset of classical AD symptoms, there is observable biological damage that produces cognitive decline [17]. Synapse loss at dendritic spines is a strong correlate of cognitive decline [18]. Synaptic dysfunction may precede cognitive dysfunction by decades [19]. Several papers have reported that synapse loss is especially prominent near mature senile plaque deposits [17-20]. In these studies, soluble $\mathrm{A} \beta \mathrm{O}$ correlates with cognitive decline symptoms. $\mathrm{A} \beta$ oligomers are found in halo regions surrounding senile plaque as well as within both pre- and postsynaptic regions in locations both near and far from plaque [19]. It has been suggested that the source 
of the $\mathrm{A} \beta \mathrm{O}$ found within the synapses in plaquefree regions is the result of $A \beta O$ migrating from the halo. There are proposals that plaques buffer the $\mathrm{A} \beta \mathrm{O}$ concentration in that they help minimize these neurotoxic compounds in vivo [16]. Synapse staining density is reported to fall off to near zero values as one approaches the Alzheimer plaque [19].

A study of these cellular oligomers has been reported [21] in which these $\mathrm{A} \beta \mathrm{O}$ molecules are classified as either Type 1 or Type 2 oligomers (Table 1). Type $2 \mathrm{~A} \beta \mathrm{O}$, the low molecular weight molecules, the predominant oligomers in the system studied, are molecules that surround senile plaque in a cross sectional halo configuration. Type 1 oligomers are comparatively few in number and found rather uniformly spread throughout the plaque-free cellular region. Type 2 oligomers have a spatiotemporal and structural relationship with amyloid plaques. They do not impair cognition in situ, possibly due to their more localized spatial sequestration around plaques. However, the much less abundant, but more widely dispersed Type 1 oligomers impair memory if extracted and tested in a mouse model, whereas Type 2 do not impair memory in the same model.

In the discussion below, this paper explains the above experimental results of these synapse-loss and alteration papers in terms of intercellular hydrological patterns and the resulting fluid shear fields from comparatively slow ISF flow through the brain parenchyma. As referenced and invoked in previous papers in this series, one added shear principle is needed in this explanation, the Metzner effect [22], namely that a shear-stretched polymer is thermodynamically driven to preferentially diffuse toward a lower shear liquid region if one is available to that polymer. The reason for the generation of Type 1 and Type 2 oligomers discussed above is attributed to differing shear types and energies generated in the two different types of brain shear fields.

\section{POSSIBLE MECHANISM FOR SHEAR-INDUCED SYNAPSE LOSS NEAR PLAQUE}

\section{Amyloid plaque origin and classification}

There are four basic subtypes of Alzheimer plaque: diffuse, stellate, classic senile, and compact [23], of which the classic senile plaque was the subject of study in synapse-loss studies discussed in the previous section. This plaque type is characterized by its solid amyloid core surrounded by a complex, mixed composition region, consisting of a more diffuse core-surrounding plaque segment that contains $\mathrm{A} \beta$ as well as neuritic process fragments. These processes are recruited to stop the growth of the central dense plaque, which they do very effectively. It is assumed that this is accomplished apparently by shielding the plaque core from flowing ISF containing shear-deformed $A \beta^{*}$, possibly converting the $A \beta^{*}$ into oligomers rather than adding it to the solid core.

Contrary to previous beliefs, the solid core in AD model mouse studies can be created and mature (cease to grow in size) in a matter of hours to days [24]. This could mean that following the establishment of an initial seed, the core must very efficiently add passing $A \beta *$ or $A \beta$ molecules until it is sealed off from ISF flow by recruited glial processes. The diffuse plaque subtype is a more open structure and is found in nearly all heathy aged individuals and apparently allows relatively free ISF flow through it. The senile (classic) plaque subtype presents a solid ISF flow impediment, somewhat like a rock surrounded by smaller rocks in a slowly flowing stream.

As discussed in previous papers in this series [4-6], it is proposed that a combination of laminar and extensional shear in such situations can distort and elongate $A \beta$ molecules and ultimately convert them, especially at higher $A \beta$ concentrations, into dimers, possibly dimer seeds [6], and resulting neurotoxic oligomers $(\mathrm{A} \beta \mathrm{O})$. However, it is also proposed that a senile plaque is not formed on the neuron surface, but in the protein structures embedded in the ISF flow channel membrane surfaces that act as ISF flow-restricting impediments [4]. Thus, the ISF shear is less than at the neuron surface, but nevertheless enough to shear-deform $A \beta$ molecules as they flow around these jutting protein obstacles and interact chemically with them, ultimately forming plaque particles from passing $A \beta *$ molecules.

\section{Shear arising from restricted ISF flow within the brain parenchyma}

In the brain parenchyma, there are many crowded objects such as neurons, astrocytes, and microglia. Many of these have large numbers of projecting processes, each one presenting an obstacle around which the ISF must flow, thus producing laminar shear.

The closer the object is to another object, the more extensional shear is generated. For example, the $\mathrm{x}$ dimension length of the arrows in Fig. 1 correspond to the relative flow distance per unit time. The shorter the arrow, the larger the shear energy 
(a)

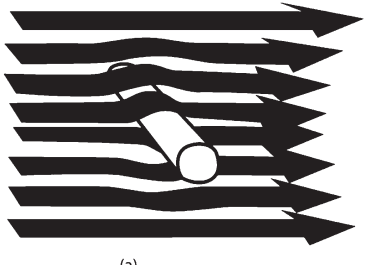

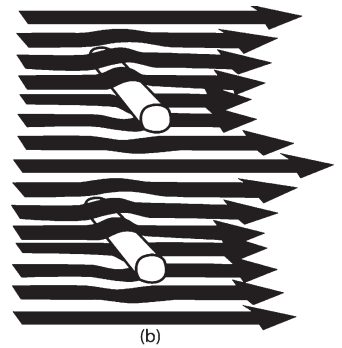

Fig. 1. Laminar and extensional flow profiles around one and two close cylindrical objects. The length of the arrows along the $\mathrm{x}$ axis indicate the forward distance travelled per unit time. The severity of arrow curvature is an indicator of the amount of laminar shear generated. In the two-object case (b), there is nearly pure extensional shear in the region halfway between the two objects.

expended in navigating around the object at different speeds in neighboring layers and in shear-deforming any molecules trapped within these layers and transforming kinetic into thermal energy.

With one object, there is only laminar shear depicted in Fig. 1(a). With two objects, Fig. 1(b), there is also extensional shear between the two flowimpeding rods, shear that is at a maximum halfway between the two rods. For example, if a dissolved molecule resides in the longest arrow path halfway between the two rods, this molecule will be accelerated much like a kayak or raft just entering rapids exactly between two large rocks in a rushing, narrow mountain stream. However, a highly flexible molecule like $A \beta$, is not only accelerated as it enters the region between the two rods-it is stretched. This is because the front part of the molecule is being accelerated faster than the rear part. This stretch is released when the front part of the molecule decelerates after it has passed the two rods. Thus, a molecule that transits through a series of "rods," e.g., neurons, astrocytes, microglia, and their processes, will be subjected to both oscillating laminar and extensional shear. It is also claimed that CSF, and possibly ISF, flows may be subjected to oscillating back-and-forth flows [25, 26], adding a possible complication to an already complicated oscillating flow and shear field.

\section{Chemical consequences of laminar and extensional shear}

As indicated above, an $\mathrm{A} \beta$ molecule under shear absorbs mechanical energy during the shearing event and forms an excited, energy-rich $A \beta^{*}$ molecule. It is shear-distorted in its molecular conformation. Because of the constricted and tortuous nature of the
ISF parenchymal flow paths, dissolved $\mathrm{A} \beta$ molecules in the brain undergo continuous cycles of shear stretching and contortion and then release from some part of these forces. Therefore, a critical question arises: Is this energy release faster or slower than the various oscillation cycles that the molecule undergoes [6]? If faster, shear energy can be absorbed and released as thermal energy with no net chemical change. If the shear energy release is slower, there must be a continuous buildup of absorbed energy, provided the molecule can absorb more shear energy. It is possible, for example, that the distorted $A \beta^{*}$ molecule has reached its stretch limit, given the maximum shear rate to which it is exposed. Thus, as it flows through the parenchyma, the $A \beta *$ molecule will contain variable amounts of shear energy. If this energy-laden molecule does not collide with another $\mathrm{A} \beta *$ molecule, it will merely cycle through various shear energy states. The net effect is to slightly heat up the solution surrounding the relaxed molecule [6] as it travels through its ISF flow path. That is, all of the shear-derived mechanical energy is converted to heat.

However, if an $\mathrm{A} \beta^{*}$ molecule collides with another $A \beta^{*}$ or perhaps a low energy, ground state (lowest possible energy at a given temperature) $\mathrm{A} \beta$ molecule, there is an opportunity to form an energetic dimer, $\left(\mathrm{A} \beta^{*}\right)_{2}$. If this dimer loses its excess shear energy as heat to its surroundings and forms chemical, hydrogen, or ionic bonds that hold the two $A \beta$ molecules together, it can become a stable, possibly neurotoxic dimer. However, it is also possible that the $A \beta^{*}-A \beta^{*}$ or $A \beta^{*}-\mathrm{A} \beta$ dimer is a seed dimer [6]. In such a case, this could initiate templated conformational change [6], similar to that found with prions [27, 28].

If the shear-excited $A \beta^{*}$ is next to or very close to the membrane surface of a neuron, then an $A \beta O$ can be formed by the high membrane surface shear, but the mechanism and biological outcome is proposed to be quite different [4]. It is suggested that most $\mathrm{A} \beta{ }^{*} \mathrm{~s}$ are those formed on or very near the membrane surface and attach to this surface, ultimately forming $A \beta O$ s on the neuron membrane surface. If enough $A \beta$ molecules coalesce, they can ultimately form a trans-membrane channel that allows $\mathrm{Ca}^{2+}$ ions to flow unhindered through the membrane, possibly fatally damaging the neuron. A recent report [29] indicates the $A \beta_{1-42}$ conformer is able to form these membrane-crossing channels, but the $A \beta_{1-40}$ conformer cannot. This is significant, since the $1-42$ conformer is much more shear sensitive than the 40 conformer. It also correlates with the fact that the 
dominant conformer in CAA deposits in the vascular system is the 40 conformer, the largest portion of which escapes from the brain parenchyma through a clearance system [30].

Given the above background, how can shearinduced chemical processes explain the recently reported results of studies of the destruction of synapses, the appearance of $\mathrm{A} \beta \mathrm{O}$ haloes around plaque particles, and $A \beta$ detected within synapses $[17,19,20]$.

\section{$A \beta O$ distribution and shear fields around plaque particles}

The following discussion will focus on possible shear effects resulting from the ISF flow slowdown caused by the presence of comparatively large cross section neuritic senile plaques in the ISF flow field. ISF flow obstacles in plaque-free regions consist of much smaller obstacles such as synapses and their associated dendrite spines. Synapses in plaque-free regions are reported to contain type $1 \mathrm{~A} \beta \mathrm{O}$ (Table 1) within both pre- and post-synaptic cells [17, 19, 20].

In both animal models and human AD patients, the density of losses of synapses and dendritic spines is reported to be greatest near senile plaques [19], leading to the hypothesis that there is a strong link between amyloid pathology and synaptotoxicity. In support of this hypothesis, Spires-Jones and coworkers $[17,19,20]$ have used array tomography [31] staining to identify the locations of $\mathrm{A} \beta \mathrm{O}$ deposits in pre- and post-synaptic cells in a mouse model and in human AD postmortem tissue. Synapse density is found to be near zero immediately next to senile plaque deposits and to increase to plaque-free zone levels beyond $50 \mu \mathrm{m}$ of the plaque edge. These senile plaques are surrounded by a cross section halo of type 2 A $\beta O$. It has been suggested $[16,12]$ that plaques are a potential reservoir of and buffer for $\mathrm{A} \beta \mathrm{O}$, which not only co-localizes with the postsynaptic density and but also whose presence correlates with spine collapse.

The discussion below suggests that the above features be attributed to liquid shear generated by the plaque's resistance to ISF flow that results in shear-induced production of $\mathrm{A} \beta \mathrm{O}$ in the immediate neighborhood of the plaque particle. In order to be as specific as possible in this discussion, the focus will be on one of the Spires-Jones group's published array tomography figures that provides a three-dimensional (3D) representation of synapse population surrounding a single plaque particle. Labels have been added

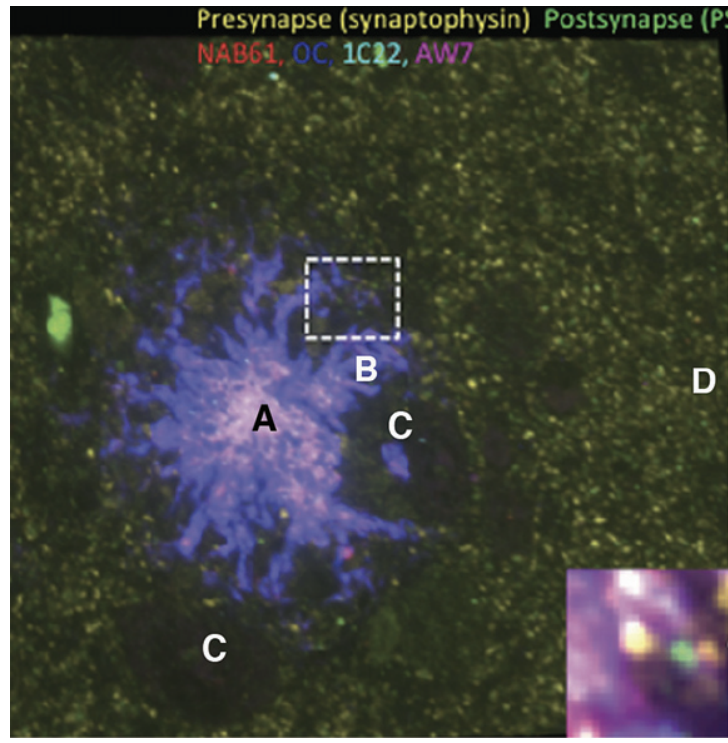

Fig. 2. Array tomography staining of brain tissue showing the presence of $A \beta$ (red, deep blue, light blue, purple antibody stains) and pre- (yellow) and postsynaptic densities (green). Letters have been added to identify regions discussed in the text. Reprinted from Pickett EK et al., Non-fibrillar oligomeric amyloid- $\beta$ within synapses, J Alzheimers Dis 53, 787-800, 2016, and modified with permission from authors and IOS Press.

to Fig. 2 to identify different regions in this figure in the discussion below.

Region "A" in Fig. 2 represents the more or less solid $A \beta$ core of the plaque. "B" represents projections from the core $A \beta$ content in which the oligomer halo is located. "C" represents two comparatively large circular regions more or less devoid of stainable synapses. "D" represents a typical synapse region far enough away from the plaque that it has about the average, more uniform, synapse density. The yellow and green spots in all regions represent pre- and postsynaptic densities, corresponding to the protein concentrations at the synaptic membranes. It is interesting that in the two roughly equal sized circular " $C$ " regions there are very few pre- or postsynapses, and that there are several smaller but similar circular regions to the right of regions $\mathrm{B}$ and $\mathrm{C}$.

\section{Proposed shear patterns around single plaque particles: Creation of the $A \beta O$ halo}

Figure 3 is a crude representation of the proposed flow patterns through a hypothetical region around a plaque particle. Ovals represent cross sections of neurons and other smaller related flow obstacles within the parenchyma near the plaque particle. 


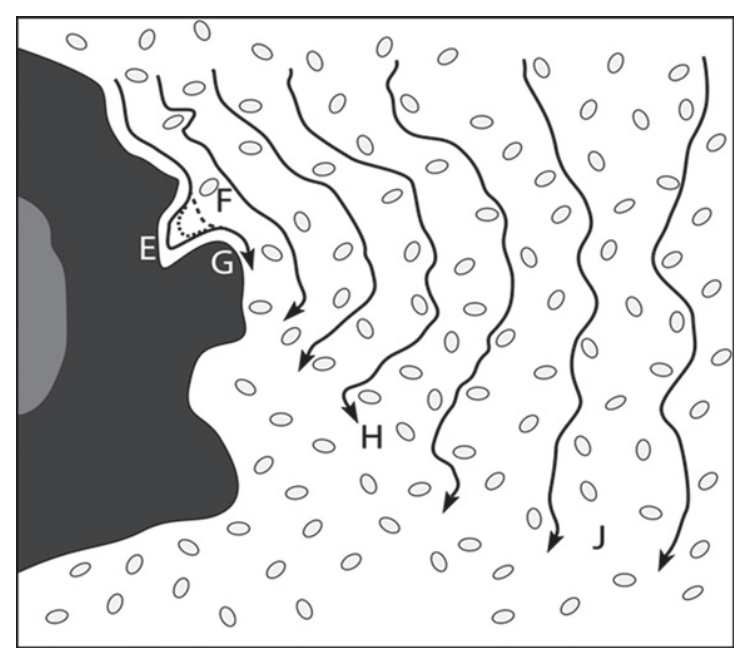

Fig. 3. Schematic of the diverted ISF flow around a senile plaque, represented on the left with a solid core and a more permeable, A $\beta O$-containing outer complex shell with projections. The length of arrows corresponds to the distance of the flow per unit time. The difference in lengths of convoluted lines predicts the regional liquid shear. Consult text for the effects of increasing ISF flow rates within the EFG pocket that lead to preferential flow of $A \beta$ into this and similar other pockets and resulting possible $A \beta O$ formation. Small ovals are crude representations of neurons and their surroundings.

Parenchymal ISF flow is obstructed by, and therefore is diverted to flow around, the solid plaque particle. This causes the flow rate to be minimum just at the particle surface because of the wetting of that surface by the ISF. In fact, the first contact layer of this fluid with the plaque particle has a zero velocity. Succeeding fluid layers next to the immobile wetted particle surface layer will have increasing, but differing, velocities, as shown in Fig. 3. This velocity difference generates a large pseudo-laminar shear field surrounding the plaque particle. This shear can distort dissolved ISF molecules such as $A \beta$, thereby generating shear-excited $A \beta *$ molecules. The lengths of black lines with arrowheads in Fig. 3 represent the distance of ISF travelled in a fixed time. This pseudolaminar shear field around plaque will be an important point in later discussions, in particular in Fig. 7.

Note that the difference between the lengths of neighboring lines decreases with distance from the particle surface. This means that the highest shear rate is immediately next to the particle surface. Therefore, the highest shear energy is next to the particle. This means that dissolved high energy $A \beta *$ molecules next to the particle surface will receive the maximum shear stretch, thus exposing hydrophobic regions of the protein, normally constrained to reside within its central regions, to hydrophilic ISF fluid. This substantially increases the propensity of these $A \beta^{*}$ molecules to dimerize if they collide with another nearby $A \beta^{*}$ molecule; the probability of this process is strongly dependent on the $A \beta$ concentration.

Figure 3 oversimplifies the shear situation in a number of ways. First, what is not shown is the "underbrush," that is, the many neuroglia and their processes, which are not represented because of their complexity, not for the lack of potential importance in flow dynamics and biological function in supporting neuronal homeostasis. Second, if the hypotheses are correct regarding the possible forward and reverse ISF cyclic flow oscillations [25, 26], the flow rates and direction of the arrows in Fig. 3 will further complicate the shear field around the plaque particle and increase the importance of the relaxation times of $\mathrm{A} \beta *$ molecules in determining the chemical consequences of liquid shear.

Figures 2 and 3 are both 2D representations of a relatively thick $3 \mathrm{D}$ slice. Figure 2 is a $2 \mathrm{D}$ reconstruction of a 3D thick cross section of the brain slice that contains a solid plaque core of $A \beta$ surrounded by broad spikes consisting of a more open mixture of $\mathrm{A} \beta$ and neurite debris. Figure 3 is a $2 \mathrm{D}$ attempt to predict a hypothetical ISF flow pattern in Fig. 2. In the proposed shear model [4], the rate of production of shear-excited states $\left(\mathrm{A} \beta^{*}\right)$ is proportional to local shear rates. The rate of production of $\mathrm{A} \beta \mathrm{O}$ would also be very sensitive in a non-linear manner to the $\mathrm{A} \beta$ concentration. Thus, at sufficiently high $\mathrm{A} \beta$ concentrations, the net production of $\mathrm{A} \beta \mathrm{O}$ should be, according to the proposed model [4], and is experimentally found to be in Fig. 2, highest next to the plaque, where the shear rate under a simpler system is highest. The yield of $\mathrm{A} \beta \mathrm{O}$ in Fig. 2 is experimentally found to drop off with distance from the plaque. Thus, this part of the model fits well with the author's anticipated shear pattern predictions found in Fig. 3.

One unique aspect of the proposed model is the shear response pattern in the typical narrow pocket in the region between " $E$ " and " $F$ " in Fig. 3. The solid, short-dashed, and long-dashed lines in the "EF" pocket, respectively, represent flow lines of ISF at comparatively low, medium, and high flow rates. Thus, the shear rates deep within the left side of the pocket leave a zone of much lower shear within the E-F pocket at high flow rates, providing there is no turbulence, which is quite reasonable given the anticipated comparatively low ISF flow rates within the ISF. In such a case, there is a pocket of low shear (E) near a region of high shear $(F)$, to which an $A \beta^{*}$ 


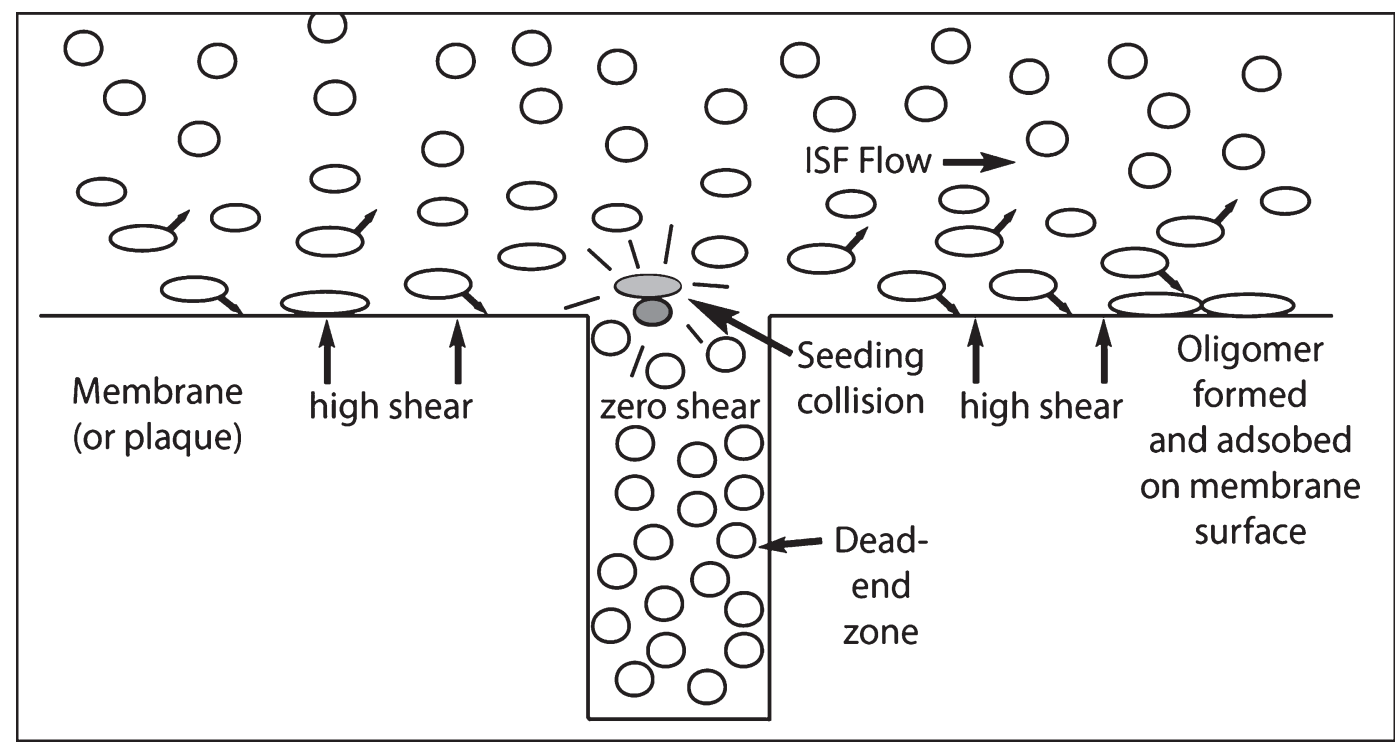

Fig. 4. Representation of a low shear zone pocket in contact with a high shear zone flow region. It also represents the shear-induced separation of molecules near the high flow zone surface, such as a membrane, into those molecules that adsorb to the surface and those that are forced to migrate in the direction of lower shear away from the surface. At the entrance to the pocket, which is a dead-end zone, a passing shear-energized molecule next to a nearby wall segment senses a lower shear zone, preferentially migrates into it [Metzner], and increases the concentrations of molecules in the dead end zone. An occasional shear-energized molecule can act as a seed that initiates a templated conformational change [6] in the dead-end zone $\mathrm{A} \beta$ molecule collection, forming $\mathrm{A} \beta \mathrm{O}$ molecules.

molecule present in the comparatively fast-flowing ISF would be exposed. According to Metzner et al. [22], in this type of situation, as the shear-stressed $\mathrm{A} \beta *$ molecule passes the $\mathrm{F}$ opening to the pocket, it will selectively and thermodynamically be induced to migrate into the E region of the pocket, also represented as the "Dead-end zone" in Fig. 4, increasing the $A \beta$ concentration within that E-F pocket.

In Fig. 4, shear-extended $A \beta *$ molecules near the shear-inducing wall, but far enough away so as not to be attracted to the wall, will be attracted toward the lower shear zone located away from the wall. This leaves a zone near the wall with lower than expected concentration because of shear-influenced diffusion away from the wall. On the other hand, because of this thermodynamically driven migration, there will be a region some distance from the wall where there is a higher than average $A \beta^{*}$ concentration that can induce $A \beta^{*}$ dimer formation, as indicated in Figs. 4 and 5 .

Increasing the $A \beta$ concentration increases dramatically the probability of forming $A \beta$ dimers, which lead to an increased rate of formation of $A \beta O$. If there is a prion-like amyloid cascade chain reaction [32] in these dead end, near zero shear, crevasses that appear to form the outer shell of the plaque particle, the many $\mathrm{A} \beta \mathrm{O}$ molecules formed from this chain would be clustered on the plaque particle surface. However, the same model process predicts that additional $\mathrm{A} \beta \mathrm{O}$ molecules would be formed in the fastest moving ISF flowing around the plaque particle edges in the highest shear zone. Thus, the combination of $\mathrm{A} \beta \mathrm{O}$ molecules formed in the crevasses and in the highest shear liquid zone around the plaque particle could appear as an $\mathrm{A} \beta \mathrm{O}$ halo in a cross section analysis of $\mathrm{A} \beta \mathrm{O}$.

However, the above-described type of seedinitiated amyloid chain reaction could also be critical to the part of the model proposed below that addresses the question, "What is the origin of the $\mathrm{A} \beta \mathrm{O}$ found within the pre- and postsynapses?" But first, let us examine the detailed effects of shear-induced migration of shear-stressed $A \beta$ away from the capillary wall.

\section{Liquid shear-induced increased concentration band above neuron surface}

The arrows attached to molecules in the layers near the surface in Figs. 4 and 5 indicate that molecules in the closest layer are attracted to the surface by mutually-attractive chemical bonds and, because of shear forces, other molecules are attracted to lower shear regions forcing them to move away from the 


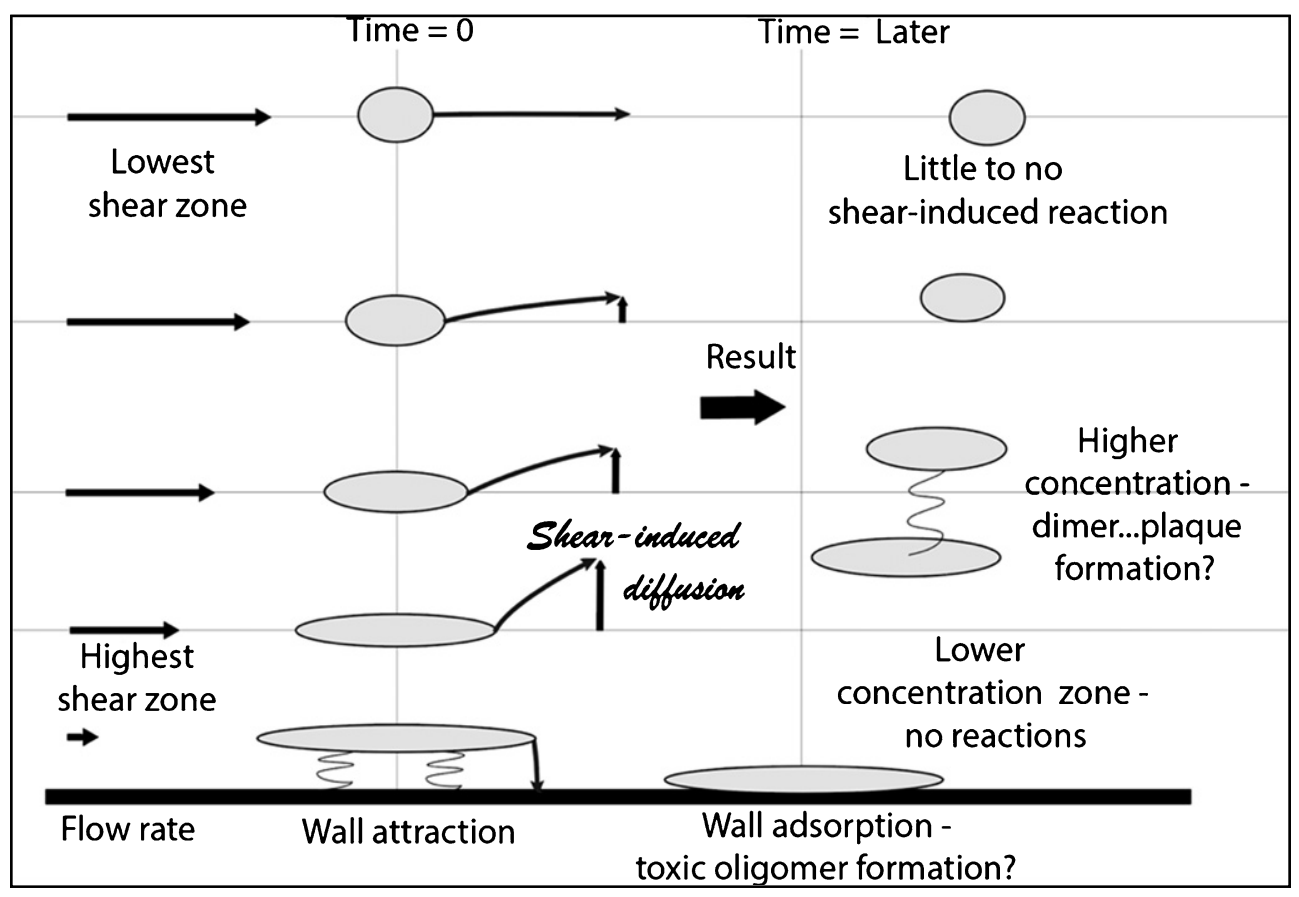

Fig. 5. Justification for dividing shear-sensitive molecules in a laminar flow situation into three groups: (a) those adsorbed to the wall; (b) those migrating away from the wall to be exposed to a lower shear rate [Metzner], increasing the local concentration of sheared molecules, thereby increasing the probability of their interacting; and (c) those that have low shear and lower concentration and therefore have fewer shear-induced reactions. "Springs" between molecules are symbols for intermolecular attraction.

surface. Figure 5 illustrates the proposed dynamic effects of forced diffusion because of laminar flow and shear-induced diffusion away from the wall. Figures 4 and 5 illustrate that, for molecules neighboring the wall and that are in a competition between shearinduced diffusion away from the wall and chemical attraction between the sheared protein and the wall, the wall wins by adsorbing this sheared protein to itself. However, it would appear from recent preliminary experiments that this protein coating is metastable (Trumbore CN, Paik J, Vachet R, unpublished results). That is, if the shear is reduced or stopped the shear-stressed adsorbed molecules relax and diffuse back into the liquid phase.

Other molecules further from the wall move away from the wall. There are two speed components, indicated in Fig. 5 by the laminar flow and shear-induced diffusion away from the wall by the same laminar shear. The net result of the shear processes, illustrated in Fig. 5, is that there is a diffuse band of higherthan-average concentration shear-stressed protein at a short distance from the protein-coated surface.

Figure 6 shows the effects of increased protein concentration on shear effects. The molecules are on average closer together, increasing the probability of dimer formation more than the doubling shown. This is a nonlinear effect. That is, doubling the concentration of molecules more than doubles the probability of formation of dimers because of this closer 3D proximity of the sheared molecules.

Because $A \beta$ is amphiphilic, as the molecule is stressed and stretched, there are wider alternating regions of hydrophilic and hydrophobic character exposed to the aqueous medium. In Fig. 6, at lower protein concentrations, the disparity in width is greater than that of neighboring stressed molecules than at the higher concentration. Thus, at the higher concentrations, attractive hydrophilic and hydrophobic forces are nonlinear functions of concentration for two reasons, closer 3-D proximity and better matching of the neighboring attractive amphiphilic regions of colliding molecules.

This shear-induced higher $\mathrm{A} \beta *$ concentration band at a short distance from the neuron or other surface is interesting because it may correspond with the distance of the bulk of the membrane-embedded proteins in such membrane surface features as the perineuronal network (PNN). These molecules also block ISF flow and may also themselves be sheardistorted. Reactions of such $A \beta^{*}$ molecules with 


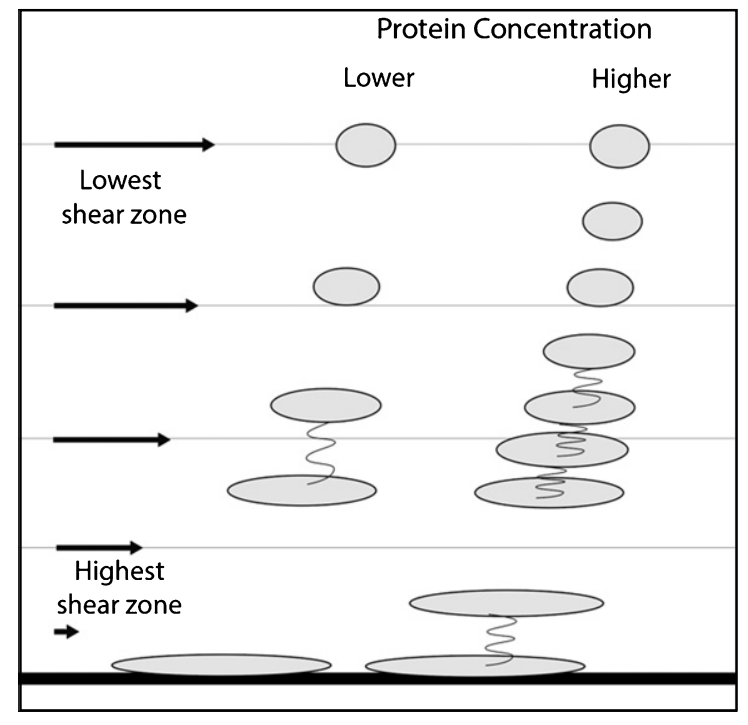

Fig. 6. The effect of changing concentration on the probability of aggregation of sheared molecules. Note that the molecules are closer together and therefore are more likely to aggregate. In a 3D situation, the aggregation probability is a non-linear function.

these molecules could well be the anchor points of the senile plaque formation. This idea is supported by the presence of structural components of PNN that are found as part of senile plaque.

\section{Shear patterns around the synapse and dendritic spines}

According to Fig. 3, every neuron close to the plaque is subject to liquid flowing around the many approximately cylindrical profiles of its projections from its soma in a flow field that is different on the right and left side of the neuron as indicated in Fig. 7c. This local asymmetrical neuronal shear field arises from the overall flow field, which is a quasilaminar field shown in Fig. 3 in which each "layer," with respect to the plaque particle, is moving with an increasing rate with increasing distance from the senile plaque particle. The upper set of arrows of different lengths in Fig. 7c indicates the differential flow rates of the ISF paths headed toward the round flow-interfering neuron segment.

Thus, since the higher flow rate is on the right side in Fig. 7c, after splitting the flow stream, the right segment of the liquid nearest the neuron will travel faster initially than the corresponding segment on the left side, causing the latter segment to accelerate to be able to meet up with that of the right side at the bottom of Fig. 7c. This acceleration causes extensional shear for any molecules contained in this left-side flow segment in addition to the laminar shear induced by the liquid forced to flow around the neuron.

Even if the flow profile is uniform, indicated by a zero shear field in Fig. 7a and b, in a region of the parenchyma which is not close to a flowimpeding plaque particle, there is laminar shear that is approximately equal on both sides of the neuron. However, at the bottom of Fig. 7a and b, there is a lower shear region where shear-distorted $A \beta^{*}$ molecules are forced by the Metzner effect [22] to migrate together, thus increasing the $A \beta^{*}$ concentration in that region where aggregation may take place between these stretched, distorted, and shear-excited monomers.

\section{MECHANISM FOR SHEAR-INDUCED PROCESSES IN PLAQUE-FREE REGIONS}

The flow-impeding objects discussed above have been approximately cylindrical. Also, the orientation of flow over the flow-impeding objects will vary. A question addressed at this point is what shear complications arise in the ISF flow patterns in plaquefree regions, e.g., around critical, different-shaped dendrites, dendritic spines, soma, and synapses in the brain? Fig. 8 illustrates some anticipated sheargenerating flowline patterns of these neural objects. Two types of dendritic spine protuberances are illustrated on the left of Fig. 8, with their altered straight-line ISF flow patterns, all of which would result in increased fluid shear on their surfaces. On the right side of Fig. 8, there is mild shear on the neuron soma surface and increased shear from the attached presynaptic segment. The middle illustration is of two of the many possible types of flowlines around a synapse. Liquid laminar shear will be generated along all of the flow lines shown in Fig. 8, and, with a high enough $A \beta$ concentration and flow rate, can produce $A \beta O$ on the membrane surface. The sheared molecules closest to the surface will be subject to membrane wall attraction and may be adsorbed by the surface, at least temporarily. Should the $A \beta$ concentration increase, this temporary attraction may turn into a more permanent dimer, ultimately leading to an oligomer as more $A \beta^{*}$ molecules are attracted to the membrane-attached $A \beta^{*}$.

However, the information illustrated in Fig. 8 is insufficient to allow an estimate of comparative shear rates. This is because nearby neighboring structures that would markedly increase the flow 


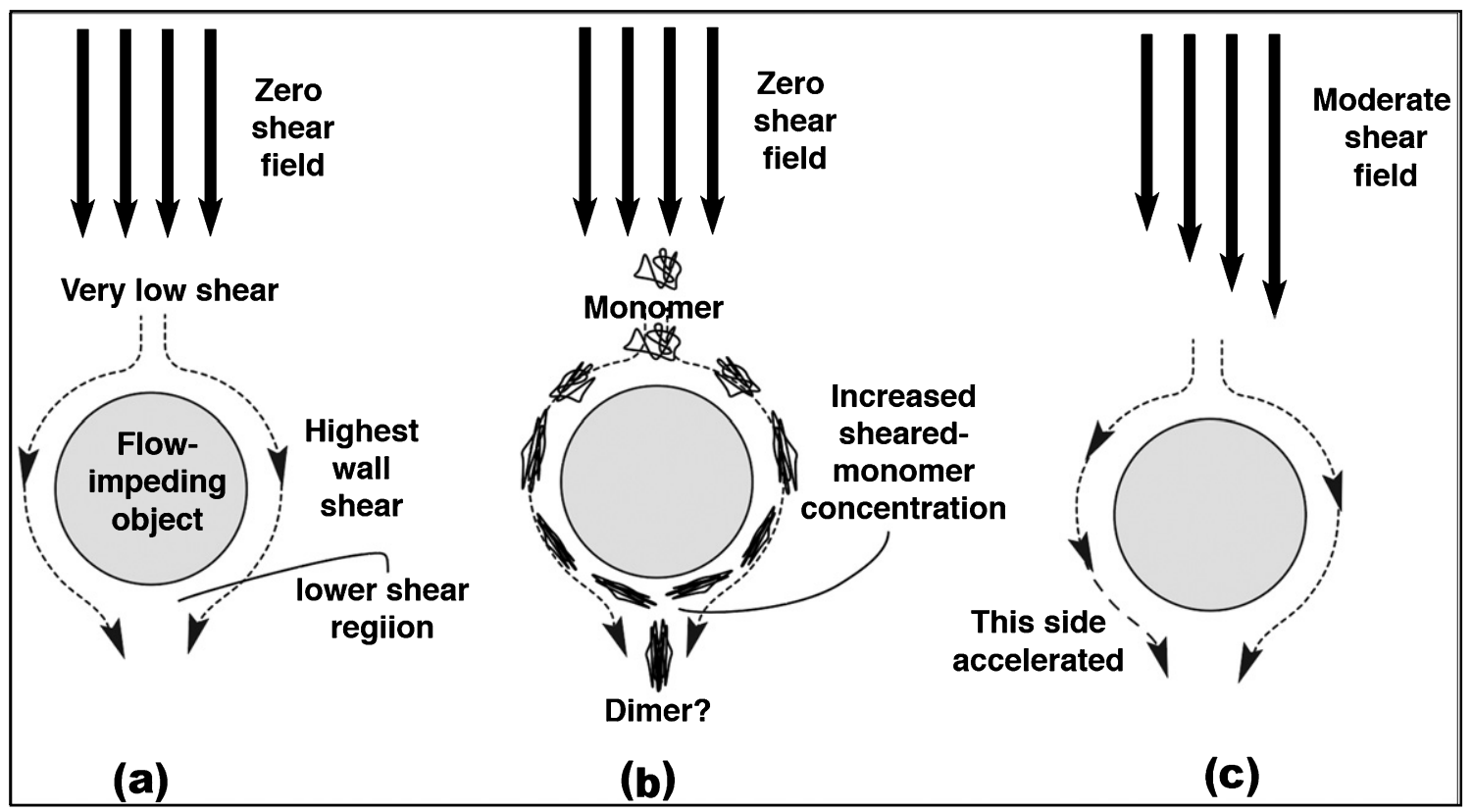

Fig. 7. Shear generated by flow around cylindrical objects in non-laminar [(a) and (b)] and laminar (c) shear fields. In (a), the uniform shear flow yields to laminar flow, with complete symmetry, thus increasing the shear distortion of polymers as they are forced to flow around the object. Shear is lower on the object's lower side, attracting molecules into this region. In (c), with a non-uniform laminar field, there is asymmetric flow, faster on the right side and initially slower on the left. However, this causes an acceleration of flow on the left side to catch up with that on the right, thus causing extensional flow that will stretch dissolved molecules on left side. The situation in (c) applies to objects near a plaque particle, where there is a large pseudo-laminar shear field developed next to the particle. Thus, additional extensional shear is generated near plaque in addition to the laminar shear developed in (a) and (b).

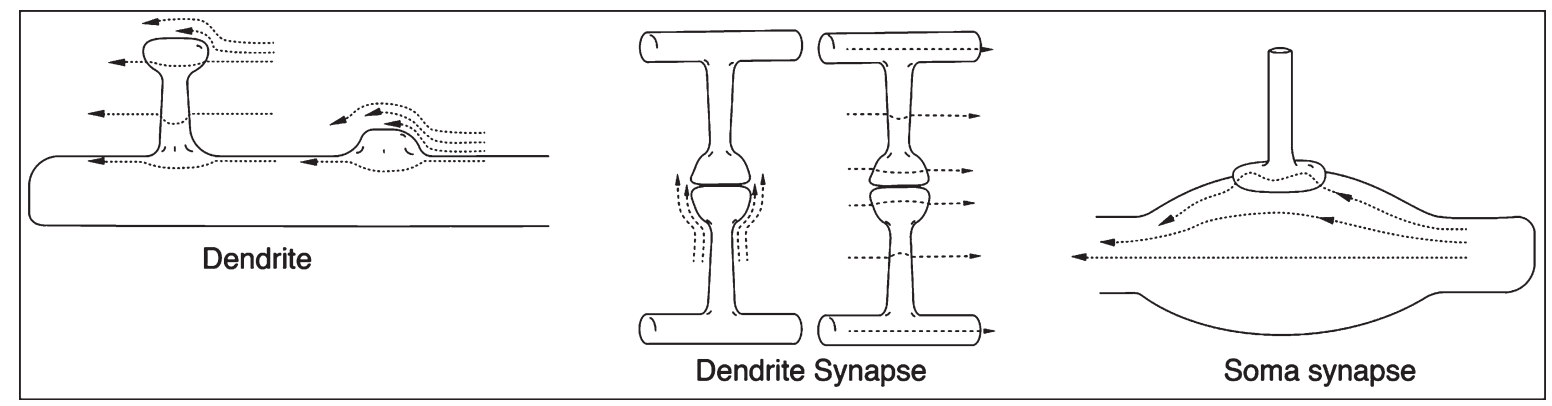

Fig. 8. Laminar flow profiles for a series of neuron and synaptic surfaces. Only one or two of the many different flow directions are shown. Differences in arrow lengths determine the shear rate differences. Not drawn to scale. Other neighboring structures such as astrocytes, microglia, etc., that will also affect the shear rates are not shown.

restrictions between all of these objects and their in vivo neighbors are not shown. Such structures would be astrocytes, microglia, other neurons, etc., and all of their numerous processes, each of which would further restrict the ISF flow. This high density of flowinterfering objects generates additional extensional shear within any fluid flowing between them. Thus, on each of these crowded surfaces there could be potential shear-induced protein aggregation processes involving $A \beta$, amyloid products, and possibly even other shear-affected proteins such as phosphorylated tau. However, parenchymal shear rates are low [4]. Thus, only $A \beta_{1-42}$ amyloid monomers, rather than $A \beta_{1-40}$ isoform molecules, are sufficiently affected by this low shear rate, providing their concentrations are high enough that two shear-excited $A \beta_{1-42}$ * molecules have sufficient shear energy to ultimately form seed dimers [6] and ultimately toxic oligomers.

The above paragraph presents a highly complex, tortuous hydrodynamic shear flow picture. There will 
be those who ask how there could be any significant ISF flow at all in this dense "neuro-jungle," and for many years the conclusion was that there was no net flow [33]. However, experiments have shown that ISF probably does flow through glymphatic brain pathways $[34,35]$. It must be noted that even very low flow rates through these very narrow confines should still generate enough shear energy to cause amyloid aggregation, at least in the case of the $A \beta_{42}$ conformer. Moreover, there are suggestions that there is a back-and-forth oscillation of CSF and possibly also of ISF $[25,26]$. If so, this cyclic movement would generate still more oscillating shear fields in most parts of the brain parenchyma, complicating already complex dynamic shear fields. However, such complexity also may offer additional opportunities for shear-energy rich $A \beta *$ molecules to relax, i.e., shed some or all of this excess energy. More experiments studying these relaxation processes are needed. Suggestions for such experiments are listed in the Supplemental Information for the second paper in this series of papers [4].

\section{Roles of astrocytes and microglia in shear-induced neuronal processes?}

The molecular and biological "underbrush" surrounding neurons, including astrocytes and microglia, tend to be under-represented both within brain illustrations and in considerations of their critical involvement with neurons. In any discussion of the role of these important neurological support and protective mechanisms in $\mathrm{AD}$, the time period of their involvement is critical. Astrocytes provide critical support for and control over neuronal activity and undergo significant changes both in pre- and postplaque periods of $\mathrm{AD}$ [36]. Upon any disturbance to brain homeostasis caused by injury in early stages of $\mathrm{AD}$ disease, microglia react in very short time intervals [37].

Before senile plaque formation, astrocytes that are affected by disease release pro-inflammatory factors and apparently lay the groundwork for the later formation of plaque. In response to these changes in the brain homeostasis, microglia, constantly monitoring the neuron and astrocyte environment, are recruited to the site of any neuron damage. Plaque with a solid A $\beta$ core apparently can form quickly in a matter of days, with the recruited microglia stopping this plaque formation by sealing the solid, approximately spherical core with a mixture of microglia and molecular debris from damaged neurons and glia. This region is the approximate location of the outer solid plaque halo that contains significant amounts of $\mathrm{A} \beta \mathrm{O}$ mentioned above. What is the possible effect of liquid shear in this region? Does ISF flow possibly generate sufficient shear to ultimately cause the formation of $A \beta O$ affecting these neural partners?

Astrocytes and microglia as well as other parenchymal constituents such as venules, arterioles, capillaries, and other microvasculature, add significant restriction barriers to the parenchymal ISF flow field. This congestion can result in significant increases in both the laminar and extensional shear encountered by dissolved ISF amyloid molecules, and result in a slowdown in the ISF flow rate. However, a significant portion of this "frictional" energy is expended in generating and maintaining laminar and extensional shear. The critical question then arises, if $\mathrm{A} \beta \mathrm{O}$ is formed primarily as a halo around senile plaque, is this the $A \beta O$ that winds up within the pre- and postsynaptic density? Diffusion through this complex tangled "jungle" might be exceedingly slow. However, it could be even slower because of adsorption of $\mathrm{A} \beta \mathrm{O}$ on these complex, possibly reactive, membrane surfaces.

\section{MECHANISM FOR THE FORMATION OF TWO DIFFERENT TYPES OF ABO}

In the experiments of Spires-Jones and her collaborators probing the $\mathrm{A} \beta \mathrm{O}$ locations in animal models and $\mathrm{AD}$ patients $[17,20]$, the speculation was that the $\mathrm{A} \beta \mathrm{O}$ found in synaptic regions migrated from the $\mathrm{A} \beta \mathrm{O}$ "halo" regions surrounding nearby Alzheimer plaque. The experimental results of Liu et al. [21] would appear to counter this suggestion since they report that the two types of oligomers, designated as Type 1 and Type 2 (Table 1), are different in biological function when extracted and injected into test animals. It is important to note that $\mathrm{A} \beta \mathrm{O}$ is reported to be present in much smaller amounts in other locations distant from plaque regions. This raises the question, is there more than one mechanism for the formation of these two types of oligomers?

The proposal in this paper is that nearly all $\mathrm{A} \beta \mathrm{O}$ found in plaque-free regions is created by milder shear-induced processes initiated by slower ISF flow throughout all parenchymal regions. If $A \beta^{*}$ molecules generated by this shear have long enough lifetimes and the local $A \beta$ concentration is high enough, it is suggested [4] that A $\beta O$ can form. However, because of the accelerated ISF flow around large 
plaque objects, the shear rates are highest next to the plaque particles, generated by the ISF flowing under quasi-laminar conditions around the plaque particles, as sketched in Fig. 3. Thus, in comparison with plaque-free regions, the regions nearest the plaque exterior should contain the highest high local concentrations of $\mathrm{A} \beta \mathrm{O}$ ("haloes") generated by comparatively high energy local shear.

Synapses can be classified for the purposes of this shear-oriented paper into two classes: those that are within the high shear zone near the plaque and those that are undergoing relatively uniform shear in comparatively low-energy shear zones distant from plaque. In the Spire-Jones et al., papers, the synapse deficit induced by the presence of plaque disappears at a distance of about $50 \mu \mathrm{m}$ from the plaque. This distance could correspond to the distance from the plaque in Fig. 3 at which the ISF flow-induced, pseudo-laminar shear around the plaque essentially disappears, as indicated by the equal lengths of the black flow lines on the right side of the diagram (Fig. 3, region F). The shear rate in this region is minimal but not zero. The question then is whether the small amount of shear in this plaque-free region is sufficient to overcome the activation energy barrier to dimer seed formation [6] when two low energy $A \beta^{*}$ molecules meet in a collision. It is suggested that $\mathrm{A} \beta \mathrm{O}$ found throughout this plaque-free region is the result of random ISF path geometries where enough shear energy is generated to overcome the barriers to $\mathrm{A} \beta \mathrm{O}$ formation.

Thus, the above discussion is an attempt to explain the role of shear in creating $A \beta O$ in both the "high shear" zone that corresponds to the "A $\beta O$ halo" zone in the Spire Jones et al. papers and the "low shear" zones at a distance from plaques. What is the source of the $\mathrm{A} \beta \mathrm{O}$ that is deposited as both pre- and post-synaptic density regions reported in these "lowshear" regions? Is it diffusion from the halo or is there another, shear-based mechanism in play?

\section{Shear-induced $A \beta O$ in pre- and post-synaptic "low-shear" regions}

Shear is suggested to be coming from two different sources: 1) the laminar macro shear pattern of flow around the plaque itself represented in Fig. 3; 2) the laminar flow of ISF over and around the neuron surface including its axon, dendrite, and soma surfaces, shown in Figs. 7 and 8. It is assumed that ISF is generating more shear in the region surrounding plaque particles than in regions greater than $50 \mu \mathrm{m}$

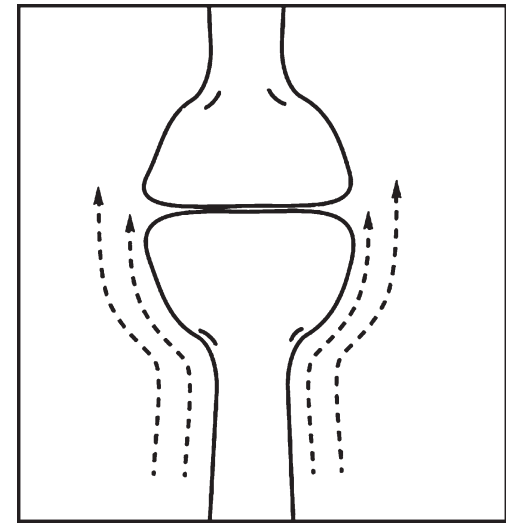

Fig. 9. Upward flow over a typical synapse. Many other flow directions are possible.

from any plaque particle. It is suggested that the $\mathrm{A} \beta O$ s that make their way into synapses in the zone near the plaque are created by the complex shear pattern, including the extra extensional shear from the pseudo-laminar flow described above. It is proposed that these have greater shear energy, generate more toxic chemical products, and thus destroy more synapses, the closer they are to the plaque particle. That is, they do not need to diffuse away from the plaque particle halo region.

But how do those A $\beta O$ s generated around those synapses at a distance from the plaque region find their way into the interior of the synapse?

Figure 9 illustrates a typical synapse's geometric structure, which governs liquid shear patterns when ISF flows over it. The synapse joins an axon presynaptic terminal process to a dendrite postsynaptic spine, forming a very narrow flat or curved synaptic cleft junction between the two terminals. The dashed lines in Fig. 9 represent a case in which flow lines are in the same direction as the axis connecting the two processes, the differences in the lengths of the two lines indicating the developing liquid shear being created along all of the membrane surfaces shown in the figure. However, there is more shear developed in the surface near the cleft because of the sudden change in diameter of the bulbous terminals that create the synapse.

Thus, a dissolved $\mathrm{A} \beta *$ molecule flowing past the cleft junction is exposed to near maximum shear in this part of its path. But the liquid contained within the narrow synaptic cleft region is in a nearzero shear region! This creates a situation much like that depicted in Fig. 4 and previously discussed, Thus, again applying the Metzner effect [22], it is 
anticipated that a passing stressed $A \beta^{*}$ molecule will selectively migrate, if possible, into the cleft, increasing the cleft $A \beta$ concentration. Increasing this concentration increases the probability of oligomer formation within the cleft-under the right conditions? But what are these conditions?

There has been speculation that the "misfolded" $A \beta$ molecule-postulated by this author to often be a shear-excited $A \beta^{*}$ with a distorted conformation [6] - is potentially able to initiate amyloid cascade reactions through a prion-like mechanism [23]. This raises the possibility that the interaction (see Fig. 4) between an $A \beta^{*}$ seed and a synaptic cleft $\mathrm{A} \beta$ molecule might spontaneously form a dimer seed and then a trimer, etc., to produce a host of $\mathrm{A} \beta \mathrm{O}$ molecules in a prion-like manner within the synaptic cleft. These molecules could then interact within the cleft uptake or synaptic receptor sites, allowing the $A \beta O$ molecules to enter both the pre- and postsynaptic densities.

There is an alternative shear-based mechanism for oligomer formation at synapses. The formation of oligomers on membrane surfaces has been discussed in a previous paper in this series [4]. The higher the shear rate of the $A \beta$-containing fluid, the higher the local $A \beta^{*}$ concentration, and the closer the shear-stressed $A \beta^{*}$ molecule is to the membrane, the greater the probability of formation of $\mathrm{A} \beta \mathrm{O}$ on (or in) the membrane surface. The geometry of the synapse, with its changing dimensions in the bulbous pre- and postsynaptic cells, could cause maximum local shear on their surfaces. The resulting surface oligomer may be able to penetrate directly into these cells and join the protein densities in the synaptic membrane.

Given sufficient conditions of flow rate and $A \beta$ concentration, shear-induced formation of $A \beta O$ might be predicted on all exterior surfaces of the synapse-as well as the surfaces of dendrite spines, as might be expected from flow lines depicted in the left illustration of Fig. 6. Early $\mathrm{A} \beta \mathrm{O}$ formation on postsynaptic dendritic spines might initiate the ultimate transfer of membrane-trapped $\mathrm{A} \beta \mathrm{O}$ molecules into the post-synaptic densities. Sufficient early deposition of $A \beta^{*}$ molecules on these spine "stub" surfaces may also prevent synapse formation.

In late stage $\mathrm{AD}$, there may be a buildup of a spine $A \beta$ solid coating that increases the diameter of the tentative post-synaptic terminals such that it cannot lengthen and pass through the holes in the PNN and therefore will not allow formation of short term memories [38]. Alternatively, the shear-induced deposition of amyloid on the PNN itself may alter the ability to participate in LTP and even cause LTD. Pickett, et al., report [19] that more $A \beta$ is found at excitatory postsynapses than at presynaptic terminals, that both pre- and postsynaptic terminals are affected by $\mathrm{A} \beta$, and that postsynaptic processes are particularly important in synapse loss. It may be that the reason is that the more rigid dendritic spines are exposed to higher shear rates for longer periods and generate more $\mathrm{A} \beta \mathrm{O}$ than the projecting presynaptic processes.

\section{Role of energy in the production of different types of $A \beta$ oligomers}

Why are the biological functions of Type 1 and Type 2 oligomers [21] in the above studies so different? The author speculates that this results from the differences in the shear rate, total shear energy, and/or types of shear involved in the flow of ISF around the flow-impeding plaque particles vs. the comparatively less massive flow interference of the many, much smaller, dendrite structures. Dendrite structures should generate smaller amounts of shear energy. However, some of this energy has to be sufficient to cause these lightly sheared $A \beta *$ molecules to seek lower shear regions near the synapse, causing an increase in the $A \beta$ concentration. As they pass by this narrow opening and come in contact with the $A \beta$ molecules within the synapse, as illustrated in Fig. 4, they can either enter the synapse or, if they have sufficient shear energy, act as seeds. If conditions are favorable, the situation is such that these seeds can form oligomers within the synapse. Because of the mechanisms present within the synapse, uptake of these oligomers molecules into both the pre- and postsynaptic junctions may be possible. Alternatively, synapse surface-shearing processes may be uniquely enhanced at low shear energies.

\section{What is the origin of the circular holes surrounding the plaque?}

In Fig. 2, there are two relatively large what appear to be holes marked " $C$ " and some other smaller circular regions containing very few stained synapses, indicating a lack of active synaptic material (ASM). It is interesting that these appear to surround or are very close to the plaque, whose arms also lack ASM in the "inlets" between these arms. A possible explanation for these ASM-depleted regions might be that they are ISF flow channels replacing eliminated ASM and due to dendritic spine shrinkage caused by 
exposure to $\mathrm{A} \beta \mathrm{O}$ [17]. It might be that these flow channels start as very small leaky flow channels that set up pseudo-laminar flow patterns in which the outer ASM walls produce high ISF shear which, in turn, produces larger amounts of $\mathrm{A} \beta \mathrm{O}$, which further inactivates and clears out more ASM debris, enlarging the holes. Some of these holes could also be indicators of unknown physiological structures, but their high concentration around the plaque seem to point to their being plaque-related and, in this model, shear-related.

The papers reporting distribution of $\mathrm{A} \beta \mathrm{O}$ around an Alzheimer plaque assume that the source of $A \beta O$ in nearby synapses is the $\mathrm{A} \beta \mathrm{O}$-containing halo and the transport mechanism is proposed to be diffusion. The greatest shear rate is found at or near the plaque or membrane surface. Therefore, if an $A \beta$ molecule is next to the sheared membrane, it is likely that it will be trapped in that surface if the $A \beta$ concentration is high enough [4]. In this case, extensive diffusion of $A \beta O$ is unlikely. In addition, both $A \beta$ and $A \beta O$ s are reported to be attracted to membranes.

If diffusion were the transport mechanism, this would not account for the fine-grain uneven distribution of synapse destruction in the immediate neighborhood of the plaque. One could not easily account for the large holes, marked "C" in Fig. 3, showing the marked absence of synapses in large, tube-like regions seen next to the plaque. Of course, one is only observing the presence of $A \beta$ and synapses and not the many other glia and other objects present in the parenchyma. However, the idea of these holes being breakthrough expanding fluid corridors carrying the ISF that is otherwise diverted around the plaque particle is quite attractive.

\section{What is the origin of the plaque?}

Two overlapping regions are suggested to be the starting and anchor points for plaque formation. One is the region above the neuron or other membrane-containing surface that contains membrane embedded proteins that extend out into the flowing ISF. These proteins can serve as both anchors for the plaque and flow inhibitors that help to shearactivate passing $A \beta$ molecules and allow them to join plaque seeds. Such seeds could be the product of higher than normal shear energy activated molecules that are able to attach to surface proteins. The second overlapping region is a region, just above but not contacting the membrane surface, that is described as a narrow layer of higher than normal $A \beta$ concentration due to shear-induced migration away from the wall. This increased concentration enhances the probability of $A \beta$ aggregation.

\section{SUMMARY}

This paper provides a hydrodynamic, shear-based mechanism for the formation of two different types of $A \beta$ oligomers produced in two different regions of both animal and $\mathrm{AD}$ patient brains, those located around senile plaque and those distributed throughout plaque-free regions. Slow glymphatic ISF is assumed within narrow, tortuous extracellular ISF channels of the brain.

One kind of comparatively high molecular weight oligomers (AßOs), designated Type 1, is produced in plaque-free regions. It is relatively uniformly distributed throughout the brain parenchyma in comparatively small amounts. Despite its small concentration, when extracted and injected into an animal model, it causes memory loss. The other comparatively low molecular weight oligomers, designated Type 2, are found surrounding senile plaque particles in a halo configuration in comparatively high amounts. Although neurotoxic to those neurons surrounding plaque particles, it does not affect memory if extracted and injected in the same animal model.

\section{Plaque-related high energy shear processes generate Type 2 oligomers}

Massive blockage of ISF micro channels by very large plaque particles cause a divergence of these micro-flows around the particle, increasing the local ISF pressure and acting as an ISF dam with diverted flow around it. The flow rate of this diverted flow is proposed to markedly increase over that in the non-blocked flow channels. The author suggests in pathology section diagrams indications of flow breakthrough channels in and around the plaque.

According to previous papers in this series, increased flow rates cause increased shear rates, which increases the probability of shear-induced dissolved $A \beta$ aggregation, ultimately leading to Type 2 A $\beta O$ formation in large amounts caused by the pseudo-laminar ISF flow around the plaque particles. The distribution pattern of shear-induced $A \beta O$ molecules around the plaque would be predicted to be a diffuse halo in cross section, much like that observed experimentally. 


\section{Plaque-free low energy shear processes generate Type1 oligomers}

ISF flow throughout the non-plaque regions is slower than around plaque, but is still able to generate shear when blocked by much smaller obstacles such as dendrite and axon projections that form synapses. Although much smaller than plaque particles, these neurites can still generate smaller amounts of shear energy than that found around plaque. However, they could still form $\mathrm{A} \beta \mathrm{O}$ through these lower energy channels, with slightly different shear-induced conformation changes and therefore expressing different biological properties of resulting $\mathrm{A} \beta \mathrm{O}$ molecules.

Laminar shear is generated by flow over these neurite surfaces. Enhanced shear is anticipated when neurite dimensions enlarge suddenly during the ISF flow path. Such enlargements occur at synapses, generating excess shear. There are two proposed types of shear processes leading to production of $A \beta O$ in the synapse vicinity:

First is the increased shear because of the bulbous nature of the pre- and postsynaptic terminals of the synapse. This causes extra shear because of both the larger surface area and fast-changing dimensions. The extra shear can cause formation of surface $\mathrm{A} \beta \mathrm{O}$ aggregates. These surface molecules may be integrated with other $A \beta$ molecules and penetrate the surface and ultimately be deposited within the synaptic densities.

The second possible $\mathrm{A} \beta \mathrm{O}$ formation process could involve a shear-activated $A \beta$ passing by the synaptic cleft and acting as a seed that, through prion-like templating, initiates the formation of $A \beta O$ s within the synaptic cleft. The synaptic cleft $A \beta$ concentration may be enhanced by the Metzner effect, which predicts that shear energized polymers undergo forced migration into zones of lower shear, if they are available, increasing the $A \beta$ concentration. The synaptic cleft is a nearly zero shear environment and would therefore attract sheared molecules. A $\beta O$ formed in this manner could utilize synaptic cleft uptake processes to enter into the synaptic terminals and deposit with either pre- or postsynaptic density at the synaptic cleft membrane.

\section{Conclusion}

Thus, the previously presented shear model [6] in addition to the above assumptions can explain nearly all of the experimental results from studies of the distribution and biological reactivity of the two types of
$\mathrm{A} \beta \mathrm{O}$ molecules and damaged synapses in the region surrounding senile plaques and in regions far from plaques. This qualitative explanation is based solely on the shear-induced formation mechanism of $A \beta O$ on critical membrane surfaces, on the hydrodynamic features created by the diverted flow of ISF around the plaque, on the relationship between shear energy and chemical outcomes of shear activated molecule reactions, and on the concept that elongated, shearstressed amyloid monomers preferentially diffuse in the direction of lower shear regions whenever possible. The above hypotheses do not preclude other possible effects of shear, such as restricting transport processes, thus enhancing shear effects postulated in this paper, as suggested by a reviewer.

\section{ACKNOWLEDGMENTS}

The author gratefully acknowledges the editorial help of Robert Suter, Elinor Thomforde, and Herbert Blades.

The author's disclosure is available online (https:// www.j-alz.com/manuscript-disclosures/17-1080r1).

\section{REFERENCES}

[1] De Strooper B, Karran E (2018) The cellular phase of Alzheimer's disease. Cell 164, 603-615.

[2] Mannini B, Chiti F (2017) Chaperones as suppressors of protein misfolded oligomer toxicity. Front Mol Neurosci 10, 98.

[3] Hane FT, Lee BY, Leonenko Z (2017) Recent progress in Alzheimer's disease research, part 1: Pathology. $J$ Alzheimers Dis 57, 1-28.

[4] Trumbore CN (2016) Shear-induced amyloid formation in the brain. I. Potential vascular and parenchymal processes. $J$ Alzheimers Dis 54, 457-470.

[5] Trumbore CN (2017) Shear-induced amyloid formation in the brain II. An experimental system for monitoring amyloid shear processes and investigating potential spinal tap problems. J Alzheimers Dis 59, 543-557.

[6] Trumbore CN (2018) Shear-induced amyloid formation in the brain: III. The roles of shear energy and seeding in a proposed shear model. J Alzheimers Dis 65, 47-70.

[7] Cohen SI, Linse S, Luheshi LM, Hellstrand E, White DA, Rajah L, Otzen DE, Vendruscolo M, Dobson CM, Knowles TP (2013) Proliferation of amyloid-42 aggregates occurs through a secondary nucleation mechanism. Proc Natl Acad Sci U S A 110, 9758-9763.

[8] Qiang W, Kelley K, Tycko R (2013) Polymorph-specific kinetics and thermodynamics of $\beta$-amyloid fibril growth. $J$ Am Chem Soc 135, 6860-6871.

[9] Trumbore CN, Tremblay R, Penrose J, Mercer M, Kelleher F (1983) Unusual flow behavior in high-performance liquid chromatography capillary tubing. J Chromatogr 280, 43-57.

[10] Hayden E, Teplow D (2013) Biophysical characterization of a beta assembly. Alzheimers Res Ther 5, 60 . 
[11] Hardy JA, Higgins GA (1992) Alzheimer's disease - the amyloid cascade hypothesis. Science 256, 184-185.

[12] Cline EN, Bicca MAB, Viola KL, Klein WL (2018) The amyloid- $\beta$ hypothesis: Beginning of the third decade. $J$ Alzheimers Dis 64, S567-S610.

[13] Ono K, Condron MM, Teplow DB (2009) Structureneurotoxicity relationships of amyloid beta-protein oligomers. Proc Natl Acad Sci U S A 106, 1474514750 .

[14] Gaspar RC, Villarreal SA, Bowles N, Hepler RW, Joyce JG, Shughrue PJ (2010) Oligomers of beta-amyloid are sequestered into and seed new plaques in the brains of an AD mouse model. Exp Neurol 223, 394-400.

[15] Ferreira ST, Klein WL (2011) The A $\beta$ oligomer hypothesis for synapse failure and memory loss in Alzheimer's disease. Neurobiol Learn Mem 96, 529-543.

[16] Brody DL, Jiang H, Wildburger N, Esparza TJ (2017) Non-canonical soluble amyloid-beta aggregates and plaque buffering: Controversies and future directions for target discovery in Alzheimer's disease. Alzheimers Res Ther 9, 62.

[17] Koffie RM, Meyer-Luehmann M, Hashimoto T, Adams KW, Mielke ML, Garcia-Alloza M, Micheva KD Smith SJ, Kim ML, Lee VM, Hyman BT, Spires-Jones, TL (2009) Oligomeric amyloid beta associates with postsynaptic densities and correlates with excitatory synapse loss near senile plaques. Proc Natl Acad Sci U S A 106, 4012-4017.

[18] Coleman PD, Yao PJ (2003) Synaptic slaughter in Alzheimer's disease. Neurobiol Aging 24, 1023-1027.

[19] Pickett EK, Koffie RM, Wegmann S, Henstridge CM, Herrmann AG, Colom-Cadena M, Lleo A, Kay KR, Vaught M, Soberman R, Walsh DM, Hyman BT, Spires-Jones TL (2016) Non-fibrillar oligomeric amyloid- $\beta$ within synapses. J Alzheimers Dis $\mathbf{5 3}$ 787-800.

[20] Koffie RM, Hashimoto T, Tai HC, Kay KR, SerranoPozo A, Joyner D, Hou S, Kopeikina KJ, Frosch MP, Lee VM, Holtzman DM, Hyman BT, Spires-Jones TL (2012) Apolipoprotein E4 effects in Alzheimer's disease are mediated by synaptotoxic oligomeric amyloid-beta. Brain 135, 2155-2168.

[21] Liu P, Reed MN, Kotilinek LA, Grant MKO, Forster CL, Qiang W, Shapiro SL, Reichl JH, Chiang ACA, JankowskyJL, Wilmot CM, Cleary JP, Zahs KR, Ashe KH (2015) Quaternary structure defines a large class of amyloid$\beta$ oligomers neutralized by sequestration. Cell Rep 11, 1760-1771.

[22] Metzner AB, Cohen Y, Rangel-Nafaile C (1979) Inhomogeneous flows of non-Newtonian fluids: Generation of spatial concentration gradients. J Nonnewton Fluid Mech 5, 449462.

[23] Delaere P, Duyckaerts C, He Y, Piette F, Hauw JJ (1991) Subtypes and differential laminar distributions of beta-A4 deposits in Alzheimer's disease - Relationship with the intellectual status of 26 cases. Acta Neuropathol 81, 328-335.
[24] Duyckaerts C, Delatour B, Potier MC (2009) Classification and basic pathology of Alzheimer disease. Acta Neuropathol 118, 5-36.

[25] Hladky S, Barrand M (2014) Mechanism of fluid movement into, through and out of the brain: Evaluation of the evidence. Fluids Barriers CNS 11, 26.

[26] Brinker T, Stopa E, Morrison J, Klinge P (2014) A new look at cerebrospinal fluid circulation. Fluids Barriers CNS 11, 10 .

[27] Colby D, Prusiner S (2011) De novo generation of prion strains. Nat Rev Microbiol 9, 771-777.

[28] Prusiner SB (1991) Molecular biology of prion diseases. Science 252, 1515-1522.

[29] Bode DC, Baker MD, Viles JH (2017) Ion channel formation by amyloid-beta(42) oligomers but not amyloid-beta(40) in cellular membranes. J Biol Chem 292, 1404-1413.

[30] Tarasoff-Conway JM, Carare RO, Osorio RS, Glodzik L, Butler T, Fieremans E, Axel L, Rusinek H, Nicholson C, Zlokovic BV, Frangione B, Blennow K, Menard J, Zetterberg H, Wisniewski T, de Leon MJ (2015) Clearance systems in the brain-implications for Alzheimer disease. Nat Rev Neurol 11, 457-470.

[31] Micheva KD, Busse B, Weiler NC, O'Rourke N, Smith SJ (2010) Single-synapse analysis of a diverse synapse population: Proteomic imaging methods and markers. Neuron 68, 639-653.

[32] Vingtdeux V, Sergeant N, Buee L (2012) Potential contribution of exosomes to the prion-like propagation of lesions in Alzheimer's disease. Front Physiol 3, 229.

[33] Wolak, DJ, Thorne RG (2013) Diffusion of macromolecules in the brain: Implications for drug delivery. Mol Pharm 10, 1492-1504.

[34] Nedergaard M (2013) Garbage truck of the brain. Science 340, 1529-1530.

[35] Iliff JJ, Lee H, Yu M, Feng T, Logan J, Nedergaard M, Benveniste H (2013) Brain-wide pathway captured by contrast-enhanced MRI. J Clin Invest 123, 1299-1309.

[36] Rodriguez J, Parpura V, Zorec R, Verkhratsky A (2016). Astrocytes in physiological aging and Alzheimer's disease. Neuroscience 323, 170-182.

[37] ElAli, Rivest S (2016) Microglia in Alzheimer's disease: A multifaceted relationship. Brain Behav Immun 55, 138-150.

[38] Tsien RY (2013) Very long-term memories may be stored in the pattern of holes in the perineuronal net. Proc Natl Acad Sci U S A 110, 12456-12461. 